\title{
Cholesterol emboli in retinal arteries
}

\author{
J. I. BALLA, J. M. L. HOWAT, AND J. N. WALTON \\ From the Regional Neurological Centre and the \\ Department of Ophthalmology, Newcastle General Hospital
}

The association of retinal artery emboli with episodes of transient blindness has been described by a number of observers. Fisher (1959) reported a patient who experienced hundreds of episodes of monocular blindness; during two of these episodes ophthalmoscopic examination revealed multiple white segments in the arteries advancing slowly along the vessels. These seemed to be micro-emboli, possibly of fibrin, occurring in association with carotid stenosis. He further reviewed the literature dating from 1870 and found 33 descriptions of the retinal circulation during transient episodes of monocular blindness. Five of these descriptions he considered corresponded closely to his own observations. Denny-Brown (1960) described similar appearances of white fibrin or platelet emboli in partially-damaged middle cerebral arteries of monkeys. Russell (1961) reported two cases of retinal emboli in association with carotid stenosis and one of these was comparable to Fisher's patient. McBrien, Bradley, and Ashton (1963) described similar findings in a post-operative case of internal carotid artery stenosis in which they were able to report on the post-mortem appearances. They found that the emboli consisted histologically of conglutinations of platelets, a few leucocytes, and a small quantity of lipid.

We have recently seen two cases of emboli of the retinal artery in which the appearances were different from those described by these authors.

\section{CASE REPORTS}

CASE 1 This patient was a 64-year-old engine driver, who two days before his admission noticed loss of vision of the right eye, which lasted for two hours. The vision then returned to normal until the morning of his admission to hospital when he had a recurrent loss of vision in the right eye, this time lasting about half an hour. On each occasion he had a sensation as if the lower lid were travelling upwards, obscuring his vision, so that with the right eye light appeared blue and he was left with a dark grey, patchy shadow before the right eye. There was no history of headaches associated with these attacks and his general health was previously good. On examination he had a blood pressure of $150 / 80 \mathrm{~mm}$. $\mathrm{Hg}$ and a soft apical systolic bruit. General examination was otherwise normal. The carotid arteries were pulsating equally, buto he had a loud bruit over the right carotid bifurcation. Visual acuity on the right was $6 / 6$ and on the left $6 / 9 . \widehat{\mathbb{D}}$ The visual fields to $1 / 330$ white target were full and the $\propto$ optic fundi normal, apart from a very small patch of exudate in the inferior temporal region of the right fundus. $\overrightarrow{0}$ Neurological examination was otherwise normal. The E.S.R. was normal, and it was thought that this was a $\vec{\omega}$ case of recurrent embolism of the retinal artery from ao stenosis of the internal carotid artery on the right side.

Shortly after admission he had a similar episode of visual disturbance; this time the visual loss affected only the lower half of the visual field and cleared up within ${ }_{\text {iv }}$ 20 minutes. During this period the retina was examined a $\rightarrow$ number of times and the only abnormal appearance was $\overrightarrow{+}$ slight segmentation in the veins. He was then giveno intravenous heparin, 15,000 units statim and 10,000 units eight-hourly. Seven hours after the first dose of heparin $\vec{D}$ the visual symptoms recurred resulting in complete bling -o ness on the right side. This began to resolve after half $\mathrm{in}$. hour, the vision returning first in the upper part of the $\overrightarrow{0}$ field with gradual recovery from above downwards. Tw hours later the improvement ceased and he was left with $a$; small central defect in the upper visual field. When the fundus was examined at this stage it was noted that there was general ischaemia, more marked in the inferioro temporal and the inferior nasal portions of the retina, with generalized oedema, and the macula showed $a \stackrel{\square}{\varnothing}$ cherry-red spot. In one of the inferior nasal arteries at its $\underset{\vec{z}}{\overrightarrow{2}}$ bifurcation there appeared a refractile bright yellow을 plaque (Fig. 1), and there was a similar plaque in the inferior temporal artery just below and temporal to the macula. The arteries beyond the site of the plaques? remained normal. He was given an injection of retrobulbar acetylcholine $(100 \mathrm{mg}$.) followed by paracentesis of the anterior chamber, but this produced no visual improve-:ment and no obvious effect on the yellow plaques. Thirty-six hours later, anticoagulant therapy was stopped, and at this stage there was no change in the appearances $\mathrm{O}$ of the fundi. Bilateral carotid angiography was then carried out and this showed bilateral carotid stenosis just? above the common carotid bifurcation with slowing of the $>$ circulation on the right side (Fig. 2). The right fundus waso re-examined half an hour after angiography and it was noted that the plaque in the inferior nasal artery had dis- $-\infty$ appeared and the plaque in the inferior temporal artery was also becoming paler. Over the next two days this plaque became gradually smaller and paler and eventually $\omega$ it disappeared. Two days later two fresh plaques appeared along the length of the inferior temporal artery, one disco 


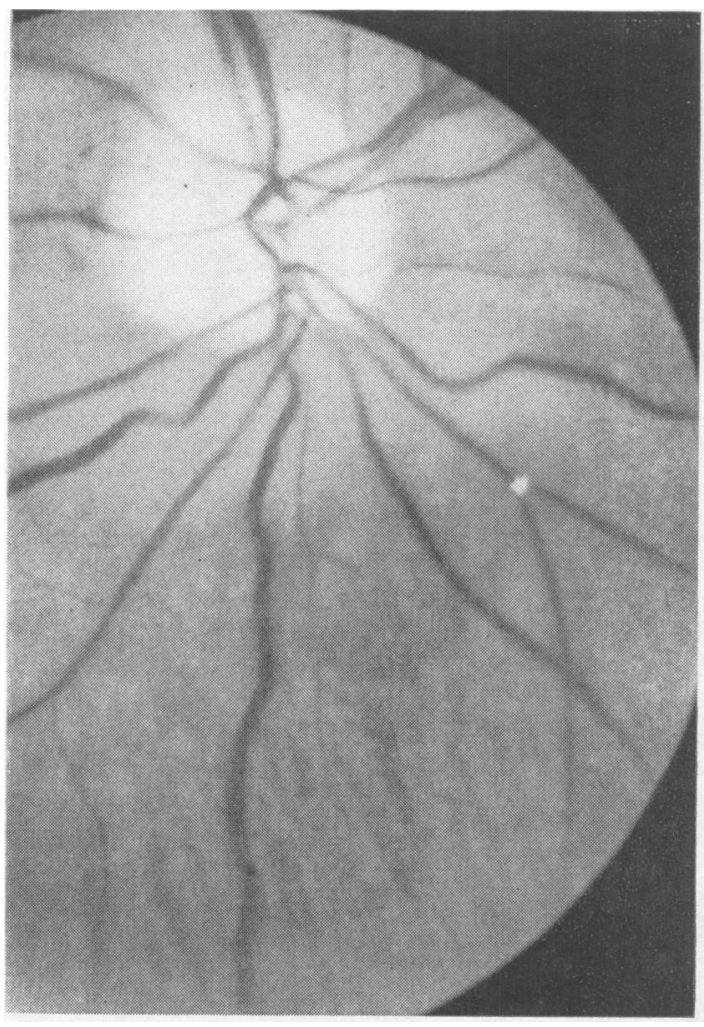

FIG. 1. Plaque at bifurcation of the inferior nasal artery.

diameter apart. These became smaller over the next two days. Right carotid thrombo-endarterectomy was then performed by Mr. J. Hankinson and Mr. P. H. Dickinson. The excised tissue was not examined histologically. Two days after operation a fresh plaque appeared at the bifurcation of the inferior nasal artery. A further two days later another plaque was observed in the inferior temporal artery, and on the next day he had an episode of blindness which lasted five minutes with complete recovery. The plaques gradually disappeared over the next few days. When seen one month later the visual acuity on the right was $6 / 18$ and the peripheral visual fields were full to $3 / 330$ white target. On the right the central visual field to $5 / 2000$ white target showed a small, dense, oval, paracentral scotoma above, bounded by the horizontal meridian below. The right optic disc appeared slightly pale, and no more plaques were observed.

CASE 2 This was a 75-year-old man who developed severe pain in the region of the right temple and behind the right eye one week before his admission. This pain persisted for a few hours and he then found that the vision in the right eye was blurred, as if a curtain had come down in front of the eye. Following the visual loss he had complete relief of pain. The 'curtain' lifted after half an hour and he could see normally again. Three days later he

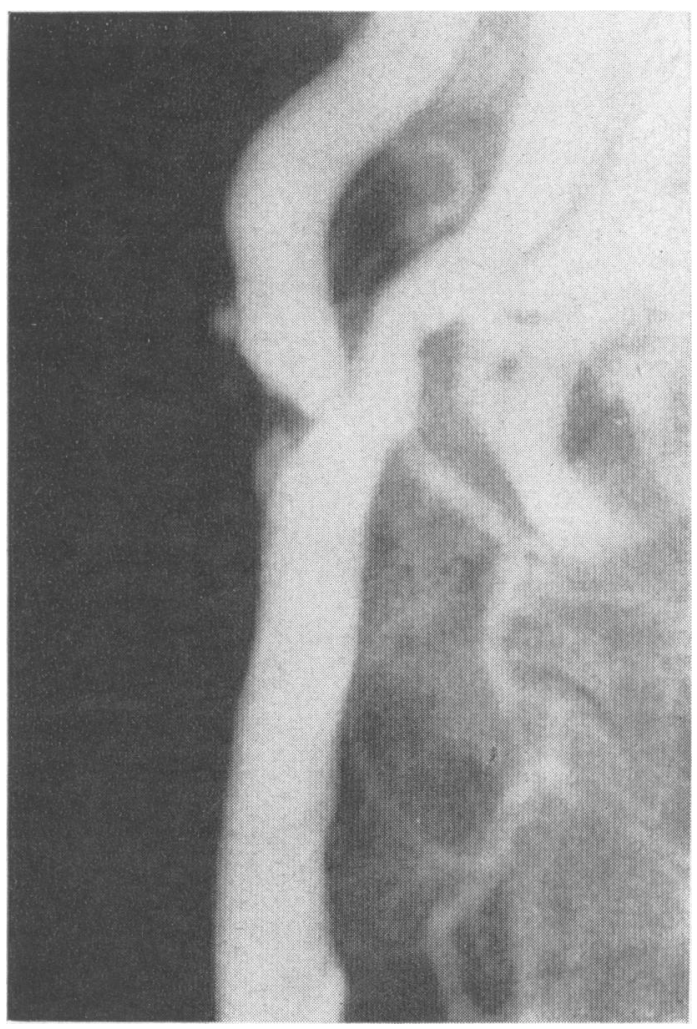

FIG. 2. Right carotid angiogram.

experienced an exactly similar sequence of events, namely, pain followed by a curtain dropping over the right eye and recovering in an hour. On the morning of his admission to hospital he once more had severe pain for a few hours followed by visual loss, which persisted for four hours. Before these episodes of visual loss his general health had been good. His blood pressure was $160 / 100 \mathrm{~mm}$. $\mathrm{Hg}$ and general examination was normal. Both carotid arteries were pulsating equally, but there was a loud bruit over the right carotid bifurcation. There was marked tenderness around the right temple but there were no abnormal pulsations of the temporal arteries. Visual acuity on the right and left corrected to 6/9. The fundi appeared normal and there were no abnormalities on neurological examination. A few minutes after admission he complained of severe pain and two hours later the curtain came down once more. About half an hour later it began to recede so that he could see objects in the superior half of the field of the right eye. On examining the right fundus half an hour after the onset of this most recent episode, refractile yellow plaques could be seen scattered throughout the arterial tree. There was one large plaque at the bifurcation of the superior temporal branch and one a little further along the same vessel (Fig. 3). There were also small sparkling, highly refractile spicules to the temporal side of the optic disc. 


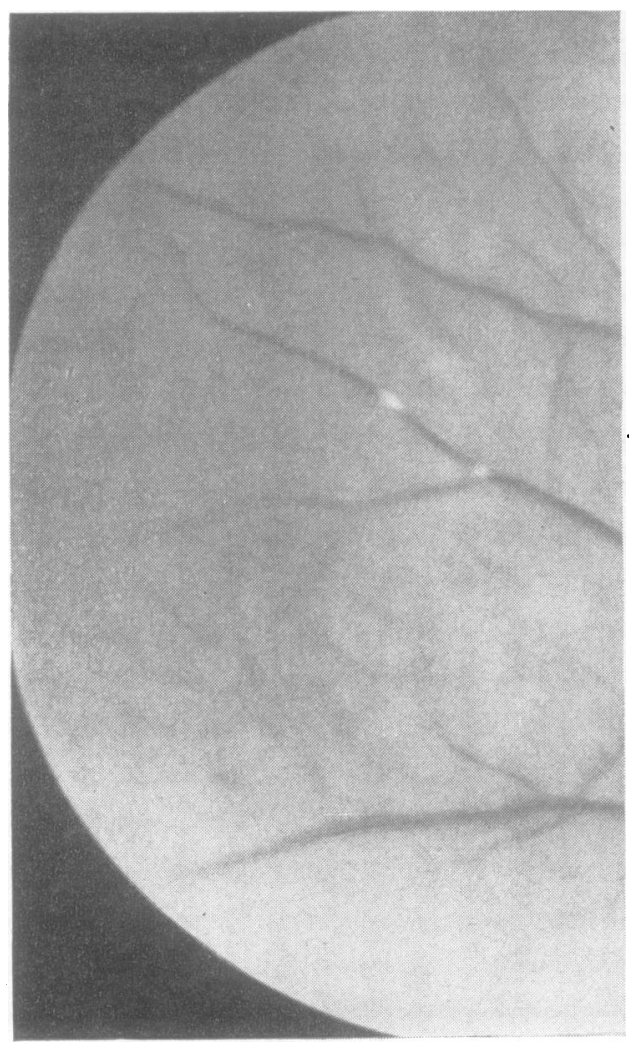

FIG. 3

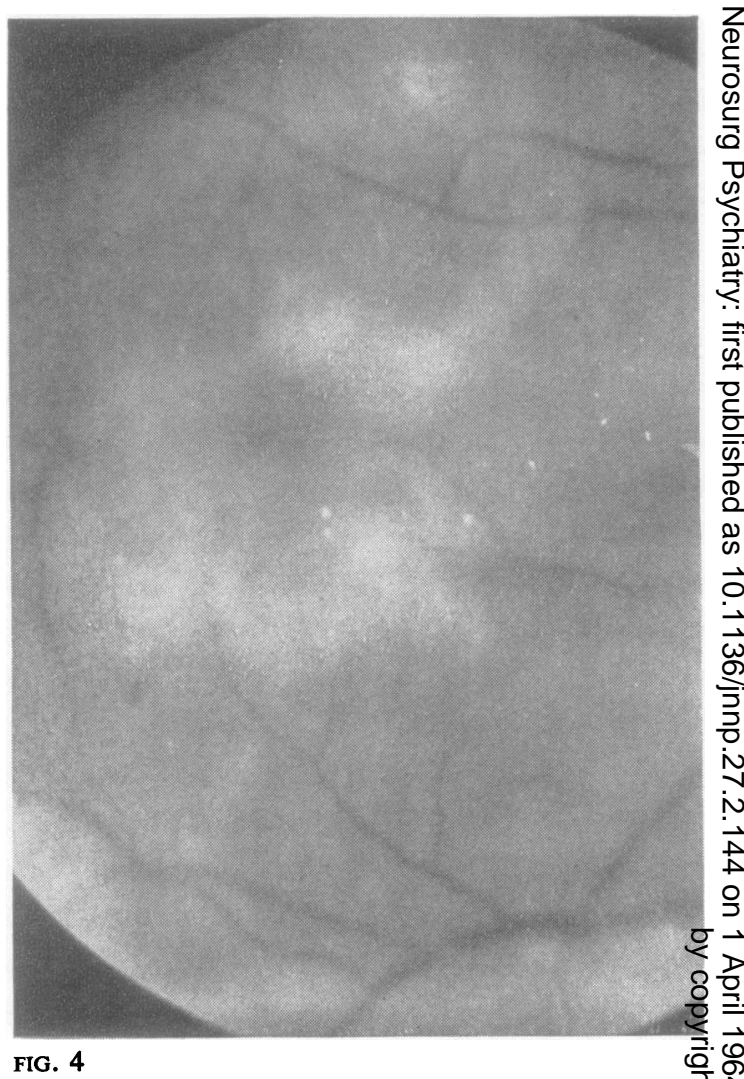

FIG. 4

FIG. 3. Plaque at the bifurcation of the superior temporal artery and one a little further along the same vessel in case?

FIG. 4. Tiny plaques distributed around the macula in case 2.

Distal to some of the large plaques the arteries appeared narrowed, but proximal to them they were normal. Circulation in the veins appeared sluggish with a suggestion of clumping. There was some oedema surrounding the superior temporal vessels and the macula was normal. Three hours later a few more bright yellow glistening spicules were to be seen in the course of the arteries. Segments of the arteries between these were apparently obliterated with surrounding oedema. An injection of retrobulbar acetylcholine $(100 \mathrm{mg}$.) had no effect. The E.S.R. and temporal artery biopsy were normal and it was thought that this patient had retinal artery emboli in association with an internal carotid artery stenosis. A radiograph of the skull showed calcification in the region of the carotid siphon. The day after admission he developed severe iridocyclitis so that it was impossible to examine the fundi for a few days, but a week after admission the iridocyclitis settled sufficiently to permit full examination of the fundi. At this stage tiny, discrete, sparkling yellow pinpoints could be seen scattered throughout the right fundus. When seen a further week later the visual acuity on the right was reduced to counting fingers. In the right fundus scintillating 'lights' were dis- tributed in a circinate ring around the macula, each lying close to the termination of a terminal macular $\mathbb{Q}$ arteriolar capillary. Few if any of these vessels were not so $\overrightarrow{\vec{A}}$ involved (Fig. 4). Beyond some of the larger plaques sheathing could be seen along the smaller radicles (Fig. 5). The optic disc was now pale.

\section{DISCUSSION}

The appearances of the optic fundi in these two cases showed a very close similarity in certain respects. The yellow plaques were extremely bright and re- 3 flected the light of the ophthalmoscope. They were multiple, tended to lodge at bifurcations, and someof them caused occlusion of the distal part of the artery, while others did not. Some tended to fade gradually; one large embolus disappeared im N mediately after arteriography (case 1) but others remained permanently sited in relation to small retinaP, arteries (case 2). Where a plaque was sited at a large bifurcation it formed the focus of a slowly develop? 


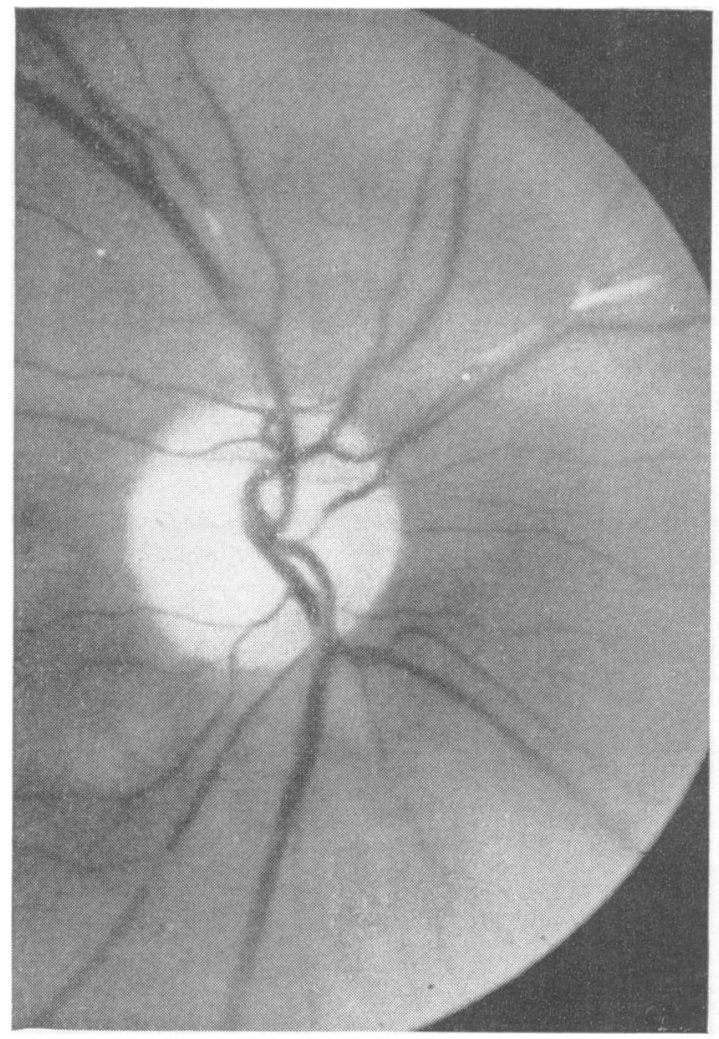

FIG. 5. Arterial sheathing spreading from the point of embolism along the superior nasal artery and the superior temporal branch in case 2.

ing process of sheathing spreading from the point of embolism along the smaller radicles distal to the bifurcation, with some exudative changes in the retina immediately adjacent to the bifurcation.

Similar appearances were described by Hollenhorst $(1961,1962)$, who suggested that these bodies were embolic crystals of cholesterol arising from ulcerating atherosclerotic plaques. He felt that the concept of cholesterol embolism was tenable since similar embolism had been reported in most other organs. It can also be reproduced experimentally, as when cholesterol material was injected into the common carotid artery of the dog by Whisnant and his colleagues (quoted by Hollenhorst, 1962). Cholesterol crystals were also reported as present in the retina in xanthomatous biliary cirrhosis by Lewis (1950), who observed scattered yellowish areas all over the fundus. She was also able to obtain postmortem confirmation (Lewis, 1952). Yellowish, glistening crystals were found around the macula in a case of familial hypercholesterolaemia by Blodi and Yarbrough (1963).

In both our cases embolism was associated with stenosis of the internal carotid artery. In the first case this was confirmed by arteriography and surgery. In the second case a loud bruit could be heard over the carotid bifurcation and there was also calcification in the region of the carotid siphon; these points, in our view, are strong presumptive evidence for the diagnosis of internal carotid artery stenosis. Hollenhorst (1962) observed these appearances in the fundi in 27 out of 235 cases of carotid artery disease and in four out of 93 cases of vertebro-basilar artery disease. He was unable to find any previous reports of similar observations.

Following the preparation of this report, the paper by David, Klintworth, Friedberg, and Dillon (1963) appeared, describing a patient who was found to have a large atheromatous plaque in the left internal carotid artery. Following surgical exploration of the artery in the neck, embolic cerebral infarction occurred in the distribution of the left middle cerebral artery. At the same time numerous shiny yellow plaques appeared in the retinal arterioles on the left side. The patient died soon afterwards; at postmortem examination it was found that the embolus in the middle cerebral artery was of the same composition as the atheromatous material in the internal carotid artery. The plaques in the retinal arterioles were also studied and were found to be composed of doubly refractile crystals containing cholesterol esters. This histological evidence, the first to be reported, would appear to lend support to our conclusions with regard to the significance of the bright retinal plaques we observed in our cases.

Though a definite relationship between the appearance of these emboli and the visual symptoms cannot be proved in all cases, there seems to be strong confirmatory evidence from the observation that certainly in the early stages of the condition the visual field defect tended to correspond to the area of retina involved by the emboli. In some cases also the emboli obviously caused actual obstruction of the arteries.

Repeated embolization of the same arteries was also observed by Hollenhorst $(1961,1962)$ and Russell (1961). Russell postulated that this may be due to propagation of a thread of thrombus from the diseased artery into one of the branches. Dollery, Hodge, and Engel (1962) have demonstrated laminar flow in the retinal circulation by fluorescent techniques. This would seem a more plausible explanation in the case of cholesterol emboli.

It would appear that cholesterol emboli may be responsible for ischaemic episodes, not only in the retinal but also in the cerebral circulation. A point 
of practical significance emerging from this observation is that anticoagulant therapy would be of no benefit in these cases. Possibly this may be one of the explanations why some patients with ischaemic episodes, presumably due to fibrin platelet emboli, are helped by anticoagulant therapy while others are not.

The distribution of these emboli in a circinate form round the macula, each related to a macular capillary vessel, would suggest a possible aetiological cause of some cases of retinitis circinata. Isolated scintillating plaques in relation to terminal arterioles are seen frequently in the course of practice, and case 2 illustrates the complete circinate distribution of emboli following one episode, or at any rate following several such episodes, over a relatively short period. It seems reasonable then to suppose that in a patient with atheromatous plaques of the internal carotid arteries, such cholesterol emboli could be given off at intervals over the years, producing in time an accumulative effect of emboli, later producing the secondary exudative changes described, finally giving the recognized clinical picture of retinitis circinata (Elwyn, 1946). It further suggests that the cholesterol deposits frequently regarded as being due to a secondary focal degenerative change in the retina in such cases may indeed be the initial emboli, the exudative changes being purely secondary in character.

\section{SUMMARY}

Two cases are reported of patients suffering episodes@ of transient monocular blindness. It is postulatedō that these were due to cholesterol emboli occurring $\square$ in association with stenosis of the internal carotid

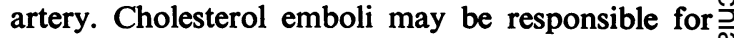
ischaemic episodes affecting the cerebral circulation, and this fact may be of significance when treatment is considered. Embolism as a possible cause of some $\overrightarrow{\bar{N}}$ cases of retinitis circinata is postulated.

We are grateful to Dr. J. B. Foster for the retinal photographs in Figs. 1, 4, and 5, and to Dr. T. Rooney for the retinal photograph in Figure 3. We would also like $\varnothing$ to thank Mr. J. Hankinson and Mr. P. H. Dickinson for $\%$ permission to quote operative findings in case 1 .

\section{REFERENCES}

Blodi, F. C., and Yarbrough, J C (1963). Amer. J. Ophthal., 55, 714. David, N. J., Klintworth, G. K., Friedberg, S. J., and Dillon, M. (1963). Neurology (Minneap.), 13, 708.

Denny-Brown, D. (1960). Arch. Neurol. (Chic.), 2, 194.

Dollery, C. T., Hodge, J. V., and Engel, M. (1962). Brit. med. J., 2, 1210.

Elwyn, H. (1946). Diseases of the Retina. Blakiston, Philadelphia.

Fisher, C. M. (1959). Neurology (Minneap.), 9, 333.

Hollenhorst, R. W. (1961). J. Amer. med. Ass., 178, 23.

- (1962). Trans. Amer. Acad. Ophthal. Otolaryng., 66, 166.

Lewis, N. (1950). Brit. J. Ophthal., 34, 506.

- (1952). Ibid., 36, 325.

McBrien, D. J., Bradley, R. D., and Ashton. N. (1963). Lancet 1, 6 Russell, R. W. R. (1961) Jbid., 2. 1422. 